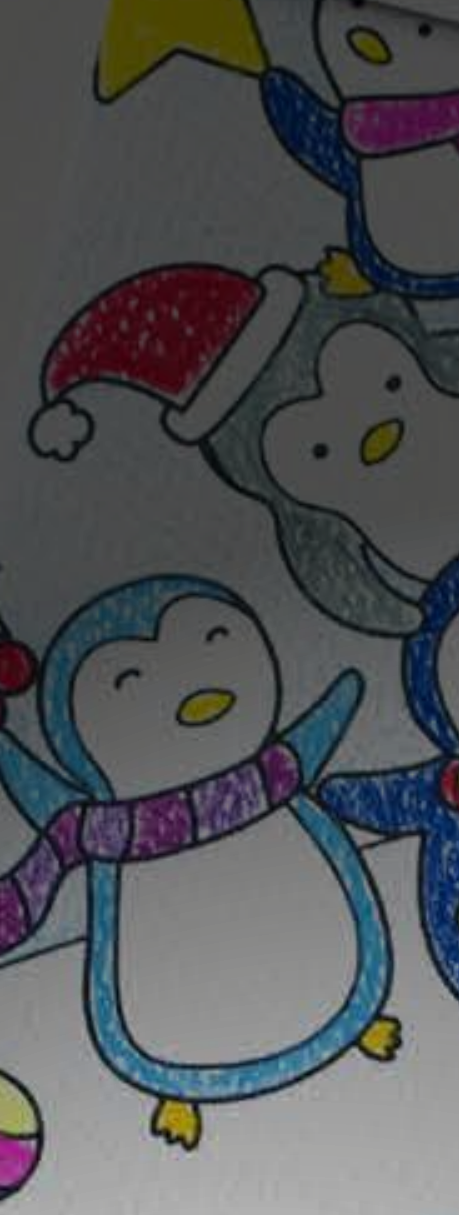
5 RevisiónlReview

són
sión Review

\title{
La educación
}

de las personas

con trastorno del

espectro autista Colombia en los últimos 10 años पT

The education of people with autism spectrum disorder: Colombia in the last

c.2 10 years

(c) $\frac{10(9)}{\mathrm{BY} \text { NC } \mathrm{ND}}$
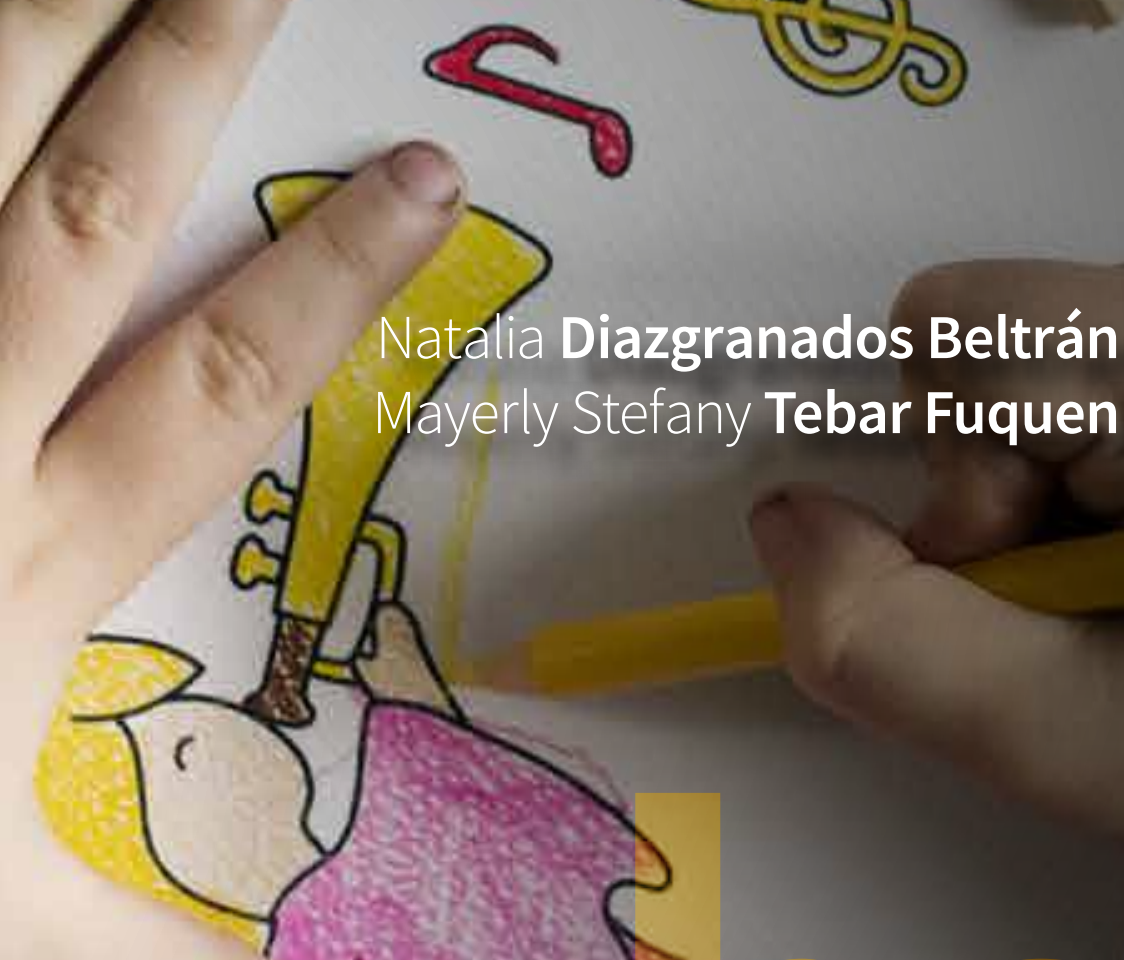

U

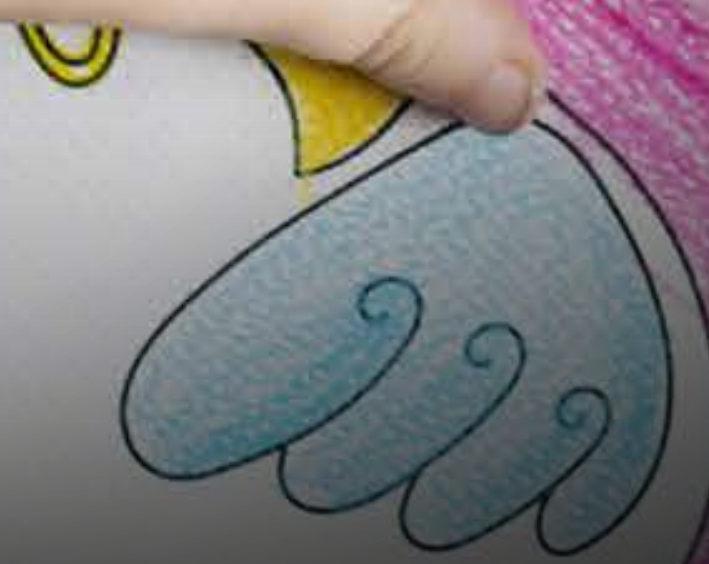

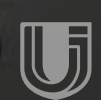

IBEROAMERICANA
HORIZONTES PEDAGÓGICOS ISSN-I: $0123-\left.8264\right|^{\text {e-ISSN }}$ 2500-705X 
ID:

0123-8264.hop. 21101

Title:

The education of people with

autism spectrum disorder

Subtitle: Colombia in the last 10 years

Título: L L educación de las personas con trastorno del espectro autista

Subtítulo: $\quad$ Colombia en los últimos 10 años

Alt Title / Título alternativo:

[en]: $\quad$ The education of people with autism spectrum disorder: Colombia in the last 10 years

[es]:

La educación de las personas con trastorno del espectro autista. Colombia en los últimos 10 años

Author (s) / Autor (es):

Diazgranados Beltrán, y Tebar Fuquer

Keywords / Palabras Clave:

[en]:

Pedagogical orientations,

educational strategies, contexts, autism spectrum disorder, population, community, family

[es]:

Orientaciones pedagógicas,

estrategias educativas, contextos, autismo,

lineamientos, Trabajo disciplinar, Población,

Comunidad, Familia.

Submited: $\quad$ 2018-07-30

Acepted: $\quad$ 2019-05-13

\section{Resumen}

El Trastorno del Espectro Autista (en adelante TEA) es una condición presente dentro de la sociedad, la cual se encuentra en constantes investigaciones y hallazgos para lograr informar a las personas sobre características presentes y metodologías que se pueden realizar dentro

de los diferentes escenarios y población. A raíz de ello el objetivo planteado se deriva de la recolección de documentos publicados en

Colombia sobre conceptos, orientaciones, lineamientos pedagógicos y estrategias

estipulados para el trabajo educativo con

las personas que presentan dicha condición durante los últimos 10 años, desde el año 2007 al 2017. Se analizaron un total de 50 documentos de este país, con los cuales se lleva a cabo un análisis descriptivo en torno a cinco categorías a) Concepto de TEA, b) Lineamientos Pedagógicos,

c) Orientaciones pedagógicas, d) Estrategias Educativas y, e) Marco normativo referente a todos los avances generados en la legislación

colombiana. Posteriormente a través de la recolección se realiza un sondeo de información proporcionada en cada año, donde destaca

significativamente el año 2017 por mayores desarrollos en torno a la problemática analizada.

Para mayor amplitud del tema, en el transcurso de la revisión se resaltan algunos documentos, con el fin de observar avances generados dentro del periodo seleccionado.
Abstract

This article derives from the collection of a state of the art about concepts, orientations,

pedagogical guidelines and strategies for educational work with people who have Autism Spectrum Disorder during the last 10 years; for this, a total of 60 documents were analyzed, from six different countries (Brazil, Spain, Peru, Colombia, Venezuela, and Cuba). The analysis gravitated around five categories a) Concept of Autism Spectrum Disorder (ASD), b) Pedagogical

guidelines, c) Pedagogical orientations, d)

Educational Strategies and, e) normative

framework referring to all the advances generated in Colombian legislation. Through

the collection, 2017 stands out as the year in which the greatest developments are observed around the analyzed problem. Subsequently, in the course of this section some of the documents

obtained are highlighted, in order to observe the development generated in the educational intervention with ASD. The study developed was documentary in accordance with the guidelines of a state of the art.

\section{Citar como:}

Diazgranados Beltrán, N., \& Tebar Fuquen, M. S. (2019). La educación de las personas con trastorno del espectro autista: Colombia en los últimos 10 años. Horizontes Pedagógicos issn-l:0123-8264, 21 (1), [pgIn]-[pgOut]. Obtenido de: https://revistas.iberoamericana.edu.co/index.php/ rhpedagogicos/article/view/1426

\section{Lic Natalia Diazgranados Beltrán}

ORCID:

0000-0003-3139-3970

Source | Filiacion:

Corporación Universitaria Iberoamericana

BIO:

Licenciada en educación especial, Corporación Universitaria Iberoamericana

City | Ciudad:

Bogotá DC [Co]

e-mail:

jdiazgranados@iberoamericana.edu.co
Lic Mayerly Stefany Tebar Fuquen

ORCID: $\quad \underline{0000-0002-2700-1885}$

Source | Filiacion:

Corporación Universitaria Iberoamericana

BIO:

Licenciada en educación especial, Corporación Universitaria Iberoamericana

City | Ciudad:

Bogotá DC [Co]

e-mail:

mtevar@iberoamericana.edu.co 


\section{La educación de las personas con trastorno del espectro autista Colombia en los últimos 10 años}

The education of people with autism spectrum disorder: Colombia in the last 10 years

Natalia Diazgranados Beltrán

Mayerly Stefany Tebar Fuquen

\section{¡Hablemos de la Educación sin barreras en la población con TEA!}

El trabajo investigativo deriva de la recolección de un estado del arte sobre concepto, orientaciones, lineamientos pedagógicos y estrategias educativas para el trabajo educativo con las población que presentan Trastorno del Espectro Autista durante los últimos 10 años en Colombia para ello, en primer lugar se desarrollan preguntas y cuestionamientos frente a los avances generados en la educación de la población con TEA, así mismo, se efectuaron búsquedas y observaciones en los diferentes escenarios a los que fue posible el acceso y la participación de las personas a nivel educativo. En segundo lugar, se da inicio a una investigación que permitiera dar cuenta de todos los avances generados en el tema, colocando una delimitación del año 2007 a 2017 para tal búsqueda.

Si bien el autismo o TEA es un tema sensible a la comunidad, esto no deja de lado la desactualización que se evidencia en la documentación y trabajo educativo. Llegando en muchas veces a la misma incógnita ¿Se Conoce lo suficiente para el trabajo educativo de la población? y irealmente existen leyes que abarquen todas las necesidades de las personas con TEA? a partir de estos interrogantes se da inicio a la recolección de documentación colombiana, con el fin de ser incentivo para que la comunidad se haga partícipe mediante la indagación, investigación, y realización de nuevos proyectos que apoyen y favorezcan a la población. 
Así mismo la desinformación se da desde inicios cuando el TEA o autismo como anteriormente se conocía, era considerado como una enfermedad ocasionada por las madres, que debido a su protección generaba un ensimismamiento en la persona Kanner (1943), posteriormente ha sido catalogado como un síndrome, discapacidad, entre otros, hasta llegar al hoy conocido Trastorno del espectro autista (TEA) el cual presenta una serie de características en las áreas de imaginación, comunicación y contexto social.

Por otro lado, desde el trabajo educativo se han evidenciado diferentes estrategias como lo son pictogramas, tableros de comunicación, llaveros, entre otros. Sin embargo, desde los resultados obtenidos se observó que se es necesario seguir desarrollando nuevos proyectosy capacitaciones que permitieran a los docentes crear nuevas estrategias educativas con la población, puesto que el escenario educativo, obliga a conocer acerca de las diferentes estrategias y orientaciones existentes.

Como último punto en el siguiente documento el lector encontrará una descripción general del rastreo histórico, estrategias, orientaciones educativas, marco normativo y los resultados obtenidos, se espera que este sea incentivo para seguir indagando y apoyando a docentes y demás comunidad. Este material permitirá revisar y comparar a profundidad todos los hallazgos y avances que han surgido a raíz de las necesidades educativas que manifiesta constantemente la Población con TEA.

\section{Evolución del Concepto del TEA}

El concepto de Trastorno del espectro Autista ha evolucionado en el transcurso de estos últimos 10 años; cambio que beneficia principalmente a la población, puesto que al ir innovando en la percepción actual de TEA es como se logran aportes relevantes en el tema de educación, lo que a su vez se refleja en estrategias, orientaciones y lineamientos pedagógicos. Partiendo de estos aportes, es cómo los docentes, padres de familia y demás comunidad, favorece a las personas con TEA, para ser partícipes dentro de una sociedad sin barreras, pensada para la accesibilidad de todos.

Ahora bien, volviendo específicamente a los cambios que ha ido teniendo el concepto TEA, es importante resaltar a Leo Kanner, quien en

3) dio a conocer a la comunidad científica, los hallazgos realizados respecto a un grupo de niños (ocho niños y tres niñas), en los cuales se evidenció una serie de características que se catalogaron como un síndrome. Dentro de éstas, se observó que se etiquetaron como débiles mentales o esquizofrénicos e incapacidad para relacionarse con pares; así mismo se les consideraba como "encerrados en sí mismos" debido a la teoría de madres nevera, puesto que se pensaba que, dada la falta de afectividad por parte de las madres, se ocasiona que el niño tuviera Autismo. Partiendo de esta teoría Kanner definió al Autismo como un trastorno de origen biológico, cuya alteración esencial consiste en una perturbación innata del contacto afectivo.

Por otra parte se encuentra (Wing, 1998) quien describe dentro del Autismo una triada, en la cual ella evidencia tres áreas alteradas en el desarrollo del ser humano, estas son: La comunicación, la imaginación y la parte social. Para lograr que la población con TEA fortalezca estos tres aspectos fundamentales descritos por Wing es importante diseñar estrategias pedagógicas que permitan desarrollar habilidades en diferentes áreas y así poder ser más independientes y autónomos dentro del contexto.
Desde el Ministerio de Salud (2015) se define TEA como un trastorno neurológico con "fuerte base genética que desde edades tempranas se manifiesta en una serie de síntomas basados en la tríada de Wing que incluye: la comunicación, flexibilidad, imaginación e interacción social" (p.29). Desde el desarrollo el TEA implica comportamientos atípicos en la interacción social, el lenguaje y en una serie de capacidades emocionales, cognitivas, motoras y sensoriales. También se dan comportamientos específicos, uno de los más característicos es alinear objetos o repetir palabras sin sentido, aunque son características que parten de los mecanismos de interacción, comunicación y pensamiento. El lenguaje, la cognición y las competencias sociales para un niño con autismo varían dependiendo la descripción del Autismo y el espectro." (MINSALUD, 2015)

De manera puntual, el término Autismo tiene una etimología griega, ya que procede de la palabra "autos" que significa "consigo mismo" , y se caracteriza por una dificultad en la adquisición de algunas áreas del desarrollo como la comunicación o la interacción con otras personas, tal y como observó Kanner en 1943 citado en (González, 2015).

\section{TEA y Educación}

El concepto de TEA es de gran utilidad en el mundo de la educación, puesto que permite entender las posibilidades y necesidades del alumnado, teniendo en cuenta las dificultades que el propio trastorno les genera. Favorece una visión integral que plantea la intervención como un reto que no solo es responsabilidad de los profesionales de la salud mental sino, y de manera muy importante, del conjunto de profesionales de la educación.

De acuerdo al (Proyecto de Ley número 046) donde se hace mención al artículo $3^{\circ}$. Definición. Entiéndase como Trastornos del Espectro Autista (TEA), al grupo de alteraciones del desarrollo en la persona, que presentan características crónicas y afectan de manera distinta a cada individuo. Se definen dentro de una disfunción neurológica crónica con fuerte base genética que desde edades tempranas se manifiesta en una serie de síntomas basados en la tríada de Wing que incluye: alteraciones en la comunicación, flexibilidad e imaginación e interacción social (p.1).

Por otra parte para el Magisterio (2017) los llamados Trastornos del Espectro Autista son perturbaciones mentales, surgidas a lo largo de la primera infancia, que afectan a menudo de forma global a la persona: la comunicación, la interacción, y a veces también las funciones psicomotoras y la maduración somática (p.73).

Con el fin de atender a las especificaciones anteriores, (2017) afirma que la tarea de los profesionales se tiene que enfocar a ensanchar las posibilidades de comunicación, facilitando el descubrimiento y el uso de los diferentes lenguajes corporales, verbales, musicales, entre otros. De esta manera se es necesario la implementación de lenguaje visual (fotografías, símbolos...), así mismo cualquier estrategia para facilitar la comunicación se vuelven necesarios para generar una participación en su contexto (p.118).

Acevedo y otros (2017) describen la triada de Wing como "triada de impedimentos" donde se describen tres áreas claves de dificultad que son las características del Autismo: a) Interacción -social b) Comunicación Social c) Imaginación Social. La tercera parte de la triada fue modificada posteriormente por algunos profesionales y se denominó flexibilidad en el pensamiento y comportamiento, lo que refleja el hecho de que el impedimento involucra la imaginación social, 
es decir, poder pensar con flexibilidad suficiente para imaginar lo que otras personas está pensando o sintiendo (p.48).

Como estrategia educativa se destaca el trabajo conjunto de padres y maestros para proporcionar las herramientas que los niños con autismo necesitan. Ya sea que estén en casa, en la escuela o fuera de casa, experimentan el mundo como un lugar confuso e impredecible. Podemos ayudar de muchas maneras:

$\rightarrow$ Crear un sentido de estructura

$\rightarrow$ Prepararlos para el cambio (la preparación previene el pánico)

$\rightarrow$ Tener en cuenta el lenguaje a utilizar previamente, puesto que en el autismo se tiende a entender todo de forma literal.

Los apoyos visuales pueden proporcionar información yestructura ayudar a la comunicación, preparar para el cambio, proporcionar tranquilidad y reducir la ansiedad y ayuda al niño al dar sentido al mundo (Acevedo, y otros, 2017). De esta manera con la información obtenida, es importante resaltar la implementación de las diferentes herramientas o apoyos para las personas con TEA con el fin de generar una mayor participación en su contexto, así pues, todas los apoyos proporcionados deberán responder a la necesidad evidenciada en las áreas (comunicativa, social, entre otras), destacando la participación que deberán tener los padres de familia durante el proceso con su hijo.

\section{Orientaciones Pedagógicas en el TEA}

Inicialmente el Ministerio de Educación Nacional crea en el año 2006 un documento con el cual se dan a conocer las orientaciones pedagógicas establecidas en Colombia para la atención educativa de la población con TEA. Se plantean una serie de orientaciones en las que se toman como referente la accesibilidad a la enseñanza- aprendizaje y capacidades del usuario.

De acuerdo con el MinEducación (2006) se deben realizar adaptaciones ambientales que se refieren a la creación de condiciones físicas de sonoridad, iluminación y ventilación en los espacios del ambiente escolar y a la accesibilidad física de la escuela y el aula. De igual manera en caso de realizar algún cambio con los muebles o elementos del aula estos deberán ser comunicados con anticipación al usuario dado que en el autismo se destaca la flexibilidad a nuevos cambios (pp. 44-45).

Siguiendo con el MinEducación (2006) se plantean las adaptaciones al currículo o elementos que permiten que los estudiantes accedan al currículo escolar. Donde se incluyen el uso de materiales especiales y sistemas alternativos de comunicación. Así mismo en los materiales se encuentran el uso de videos, dibujos, láminas, pictogramas, entre otros. Permitiendo que el usuario culmine su proceso educativo además de tener una participación activa en contexto escolary social (p.46).

Por tal motivo partiendo de las características de cada población y más aún de cada persona, se expide el Decreto 1421 de (2017), en donde se hace referencia a los ajustes razonables como aquellas acciones, adaptaciones, estrategias, apoyos, recursos o modificaciones necesarias y adecuadas del sistema educativo y la gestión escolar, basadas en necesidades específicas de cada usuario. A través de estas se garantiza que puedan desenvolverse con la máxima autonomía en los entornos en los que se encuentran, y así poder garantizar su desarrollo, aprendizaje y participación, para la equiparación de oportunidades y la garantía efectiva de los derechos (p.4).
Así mismo se destaca que la permanencia educativa para las personas con discapacidad, comprende las diferentes estrategias y acciones que el servicio educativo debe realizar para fortalecer los factores asociados a la permanencia y el egreso de los niños, niñas, adolescentes, jóvenes y adultos con discapacidad en el sistema educativo, relacionadas con las acciones afirmativas, los ajustes razonables que garanticen una educación inclusiva en términos de pertinencia, calidad, eficacia y eficiencia y la eliminación de las barreras que les limitan su participación en el ámbito educativo (MinEducación, 2017, pág. 5).

De acuerdo con lo anterior se evidencia la necesidad de implementar estrategias que promuevan en los diferentes contextos la participación activa de cada una de las personas con discapacidad, brindando los respectivos apoyos de acuerdo a las características de cada uno.

Retomando el Decreto 1421, respecto a su implementación en los diferentes escenarios educativos, se ha fijado un plazo, el cual vence hacia finales del año, en donde todas las instituciones educativas deberán contar con los ajustes razonables de acuerdo al usuario que lo haya requerido.

Como último punto, se resalta la importancia de contar con documentación actualizada, con el fin de proporcionar una mejor intervención pedagógica Específica para la población con TEA, así como la implementación de nuevas estrategias de acuerdo a los diferentes contextos que se presentan.

De igual manera siguiendo lo expuesto en el Congreso de la República (2017), desde la parte educativa se establecen algunos de los siguientes criterios:

a. La obligación de las secretarías de educación distrital o municipal, de asignar cupo a la persona con TEA, en la institución educativa pública o privada bajo la modalidad de convenio, en la que exista programa de inclusión para personas con TEA

b. Que la institución educativa asignada, a través de los profesionales de apoyo realicen la evaluación pertinente a la persona con TEA, para determinar: Nivel o grado de escolaridad al cual ingresa el alumno; fortalezas y necesidades para proyectar flexibilización curricular o Si el alumno necesita acompañamiento en aula regular.

c. Definir qué profesionales podrán prestar sus servicios de prácticas, como acompañante pedagógico en aula regular, la forma o convenio institucional por parte de las universidades con las secretarías de educación distrital y municipal para tal fin

(Congreso de la Republica, 2017).

\section{Estrategias Educativas}

Según Bravo (2008) las estrategias educativas son todas las acciones realizadas por el docente, con el fin de facilitar la formación y el aprendizaje de los estudiantes. "Componen los escenarios curriculares de organización de las actividades formativas y de la interacción del proceso enseñanza y aprendizaje donde se logran conocimientos, valores, prácticas, procedimientos y problemas propios del campo de formación" (p.52).

A continuación, se resaltan algunas estrategias educativas.

Protocolo clínico para el diagnóstico, tratamiento y ruta de atención integral de niños y niñas con Trastornos del Espectro Autista. 


\section{Metodología ABA}

De acuerdo con el (MINSALUD, 2015) la metodología ABA fue utilizada en un principio como una técnica de enseñanza utilizada para trabajar con población autista y estuvo basada en el modelo conductista que luego se convirtió en lo que hoy se conoce como ABA, con el tiempo esta técnica fue evolucionando y se incorporaron otras estrategias de la psicología cognitiva y el procesamiento de información. Así mismo, fue desarrollada por Ivar Lovaas, donde fue enfocada al estímulo del ambiente y el comportamiento de la persona, para fortalecer o educar en diferentes áreas como: académicas, sociales, y reducción de comportamiento no deseado. Esto se logró por medio de trabajos específicos en sus habilidades de comunicación (p.41).

El enfoque en este programa se basa en la capacitación de tareas discriminadas, utilizadas para reducir comportamientos disruptivos con la estimulación de comportamientos socialmente aceptados. La intervención se puede iniciar en niños de 3 años de edad y dura de 2 a 6 años. De igual manera, el avance se mide en consecución de metas individuales y no en el tiempo de estancia en el programa de un usuario. En la metodología ABA se realiza un trabajo de siete días a la semana con sesiones de 5 a 7 horas totalizando un promedio de 40 horas semanales, a su vez, cada tarea específica tiene tres componentes (verbal, gestual y físico), los cuales buscan hacer más probables los comportamientos deseados, para que entonces puedan ser reforzados.

Por otro lado, el instituto Lovaas, en los procedimientos de ABA, trabaja uno a uno con padres de familia, buscando establecer una buena relación y desarrollar relaciones constructivas y positivas como

(MINSALUD, 2015, pág. 42)

$\rightarrow \quad$ Interacciones positivas: se desarrollaron por primera vez a través del uso de las actividades favoritas y la respuesta a cualquier intento de comunicarse.

$\rightarrow$ Motivación: se fomenta a través de la utilización de los materiales familiares y refuerzos específicos para los niños.

$\rightarrow$ Participación: de los padres es crítica. Los padres tienen el poder a través de la capacitación y la colaboración para crear un entorno en el que se proporciona tratamiento de la mayor parte de las horas de vigilia de un niño, en el hogar, en la escuela y en el vecindario.

$\rightarrow$ Aprender a hablar y entender el lenguaje vocal se considera una parte fundamental del desarrollo social. Imitación también es crucial, ya que permite a un niño a aprender observando a otros niños a aprender.

\section{Sistemas Aumentativos y Alternativos}

Acorde con Almazán Martínez (2009) los sistemas de comunicación son todos aquellos recursos o técnicas que permiten la expresión y comprensión diferente a la palabra hablada.

Así mismo los sistemas alternativos y / o complementarios de comunicación pueden dividirse en dos grandes grupos (Almazán Martínez, 2009, pág. 1 :

$\rightarrow$ Los sistemas con instrumentos, como pueden ser: fotografías, dibujos, objetos, pictogramas, entre otros elementos, entre los que podemos encontrar, sistemas como el sistema de palabra complementada (SPC) o el sistema Bliss.

$\rightarrow$ Los sistemas que no precisan ayuda de instrumentos, como, el lenguaje de signos, bimodal, cued-speech, ya que el medio de expresión son los gestos, y no precisa de ninguna herramienta, tan sólo precisa de los gestos realizados por la persona que participa en el proceso de comunicación.

Es así cómo podemos definir la comunicación alternativa como cualquier forma de comunicación distinta del habla y empleada por una persona en contextos de comunicación. Ejemplo: El uso de signos manuales y gráficos, el sistema Morse, la escritura... son formas alternativas de comunicación para una persona que carece en la habilidad del habla, por otro lado, la Comunicación aumentativa significa comunicación de apoyo o de ayuda. La palabra aumentativa subraya el hecho de que la enseñanza de las formas alternativas de comunicación tiene un doble objetivo: promover y apoyar el habla, y garantizar una forma de comunicación alternativa si la persona carece de esta. Von y Martisen, 1993 citado por Almazán Martínez (2009)

A continuación, se nombrarán algunos sistemas de comunicación aumentativa y alternativa (Almazán Martínez, 2009, págs. 4-5):

\section{Pictogramas Ideográficos de Comunicación}

Consiste en dibujos blancos utilizados sobre fondo negro y la palabra escrita en blanco. Combina símbolos pictográficos e ideográficos.

Posibles usuarios del sistema:

$\rightarrow$ Personas con limitaciones en la comunicación oral.

$\rightarrow$ Adecuado para ciertas personas con discapacidad visual.

\section{Símbolos Pictográficos para la Comunicación}

Es un sistema basado principalmente en símbolos pictográficos (se complementa con ideogramas y abecedario).

Actualmente, es uno de los más utilizados en comunicación aumentativa y alternativa.

\section{Características del SPC:}

$\rightarrow$ Representan las palabras y los conceptos más habituales en la comunicación cotidiana.

$\rightarrow$ Sencillez en cuanto al tamaño.

$\rightarrow$ Universalidad (adaptado a múltiples idiomas.)

$\rightarrow$ Se ofrece en un soporte reproducible con facilidad.

$\rightarrow$ Los símbolos se proporcionan tanto en blanco y negro como en color, con fondo o reborde de color.

\section{MINSPEAK}

Tiene un soporte electrónico o dispositivo que tiene salida de voces digitales, sintetizadas o ambas a la vez, almacena esta información. A su vez tiene su origen en el lenguaje de los jeroglíficos y está diseñado para una serie de comunicadores con voz.

\section{Arte-Terapia como sistema de comunicación}

Mediante la investigación de Beltrán, Guzmán, Mazo Pineda, Segura Sila y Tapias (2016) se observa que desde la arte-terapia se puede diseñar un sistema de comunicación para la población con TEA. Esto se afirma ya que según la Asociación Chilena de Arte Terapia citado por Beltrán, Guzmán, Mazo Pineda, Segura Sila, y Tapias (2016) es una especialización profesional que aprovecha sus distintos modos para la expresión, para lograr que el niño, joven y adulto puedan comunicarse 
por medio de dibujos, arte plástica, musicoterapia, entre otras y así saber y establecer el estado en el que se encuentran con un fin de desarrollo personal y educativo (pás

Por medio de los hallazgos encontrados se establece como una forma de expresión saludable y creativa, que en el caso de la persona con autismo juega un papel importante ya que ellos al no tener una forma de expresión adecuada para una comunicación con su entorno, pueden decir o expresar de una forma inventiva pensamientos o sentimientos que tengan. Además, es una forma adecuada de que mejore su ánimo, reduce el estrés y una facilidad de comunicación con sus familiares y quienes les acompañan en su entorno, esa es una estrategia por medio de un dibujo. (Beltrán, Guzmán, Mazo Pineda, Segura Sila, \& Tapias, 2016).

Por otra parte, la musicoterapia facilita la comprensión y aprendizaje al asociar la música con temas determinados lo cual es más fácil de asimilar para los autistas ya que si se le llega a olvidar algo, bastaría con recordar la canción para relacionarla con el contenido. En relación a lo anterior es conveniente que las canciones sean presentadas de manera clara y lenta. "La musicoterapia permite también que el niño autista descubra su cuerpo y el espacio que lo rodea" (Vaillancourt, 2012, pág. 116).

\section{Marco Normativo}

Actualmente desde la legislación colombiana el TEA está clasificado dentro de la población con discapacidad, a raíz de ello los decretos y leyes establecidos en los últimos años son creados para respaldar y proteger a la población en general. A continuación, se analizan todas las Leyes y Decretos estipulados en Colombia para la atención y protección de la población.

\section{Ley General de Educación 115 de 1994}

Pormedio delaley 115 de (1994) sereglamenta quetodaslasinstituciones educativas deberán realizar la adaptación correspondiente al currículo cuando se evidencie que el estudiante lo necesite o de presentar alguna discapacidad. Así mismo las instituciones educativas contarán con capacitaciones que le permitan guiar el trabajo o intervención con determinada población con discapacidad.

\section{Ley 1145 de 2007}

Mediante la Ley 1145 (2007) se manifiesta la participación activa en el proceso para la formulación de la Política Pública para la Discapacidad, en el marco de los derechos humanos. Para tal propósito se solicita Presentar recomendaciones técnicas para el desarrollo de la política social a favor de las personas con algún tipo de discapacidad, esto con el fin de poner en marcha políticas, planes, estrategias y programas de intervención del sector de la discapacidad.

\section{Decreto 366 de 2009}

Por medio del Decreto 366 (2009) se reglamenta los servicios de apoyo pedagógico para personas con discapacidad, este será aplicado a las entidades territoriales en la organización del servicio de apoyo pedagógico para la oferta de educación inclusiva a los estudiantes que encuentran barreras para el aprendizaje y la participación por su condición de discapacidad y a los estudiantes con capacidades o con talentos excepcionales, matriculados en los establecimientos educativos estatales.
De igual manera los establecimientos educativos deberán organizar, flexibilizar, y adaptar el currículo y el plan de estudios, conforme a las condiciones y estrategias establecidas en las orientaciones pedagógicas producidas por el Ministerio de Educación Nacional.

\section{Ley Estatutaria 1618 de 2013}

Principalmente en escenarios educativos las personas con TEA están amparadas desde la Ley Estatutaria "1618" (2013) que manifiesta en el artículo 11 el derecho a la educación y atención a la población con necesidades educativas especiales, fomentando el acceso y la permanencia educativa con calidad, bajo un enfoque basado en la inclusión del servicio educativo.

En consecuencia, el Congreso de Colombia (2013) debe "crear y promover respeto a la diversidad desde la perspectiva de los niños, niñas y jóvenes con necesidades educativas especiales, como sujetos de derecho, específicamente su reconocimiento e integración en los establecimientos educativos oficiales y privados".

Garantizar el derecho de los niños, niñasy jóvenes con necesidades educativas especiales a una educación de calidad, definida como aquella que "forma mejores seres humanos, ciudadanos con valores éticos, respetuosos de lo público, que ejercen los derechos humanos y conviven en paz. Una educación que genera oportunidades legítimas de progreso y prosperidad para ellos y para el país. Una educación competitiva, que contribuye a cerrar brechas de inequidad, centrada en la Institución Educativa y en la que participa toda la Sociedad".

\section{Decreto 1421 de 2017}

Actualmente se establece el Decreto 1421 (2017) en donde el estudiante con discapacidad que se encuentra en proceso de ingreso al sistema educativo formal deberá "contar con diagnóstico, certificación o concepto médico sobre la discapacidad emitida por el sector salud y con el PIAR o el informe pedagógico si viene de una modalidad de educación inicial, que permita identificar el tipo de discapacidad". (MinEducación, 2017) En caso de que el estudiante no cuente con dicho requisito, se deberá proceder con la matrícula y con el registro de las variables para la identificación de los estudiantes con discapacidad en el SIMAT, con base en la información de la familia y se efectuará el reporte correspondiente a la respectiva secretaría de educación, o entidad que haga sus veces, para que en articulación con el sector salud se establezca el diagnóstico y el proceso de atención más pertinente, en un plazo no mayor a tres meses.

Esta intervención se implementa con el fin de garantizar una educación de calidad, haciendo valer los derechos de todas las personas, además de brindar alternativas en el acceso a las instituciones o en su defecto fundaciones.

Dentro de esta línea se retoma el Decreto 1421 de (MinEducación, 2017) donde se presenta el Plan Individual de Ajustes Razonables PIAR (2017), definido como todas aquellas acciones, adaptaciones, estrategias, apoyos, y recursos necesarios y adecuados del sistema educativo y la gestión escolar, basados en las habilidades de los usuarios, además, se garantiza que puedan desenvolverse con la máxima autonomía en los entornos en los que se encuentran, y así poder garantizar su desarrollo, aprendizaje y participación. (p.3)

Así mismo se tiene en cuenta la participación activa que deben tener las familias de los usuarios en su proceso escolar, consolidando 
también las acciones o compromisos que establecen las familias como actores corresponsables en la educación de sus hijos con diversidad funcional.

Dentro de los ajustes razonables (2017) se encuentra la realización de actividades, materiales, espacios, apoyos para facilitar la comunicación y apoyos en el proceso de aprendizaje y todos aquellos que el docente de aula, docente de apoyo, docente orientador y otros profesionales consideren necesarios para lograr la participación y proceso de aprendizaje en el usuario. (pp. 2-4)

\section{Proyecto de ley 046}

Mediante el proyecto de ley 046 de (2017) se establece que la institución educativa asignada, a través de los profesionales de apoyo realicen la evaluación pertinente a la persona con TEA, para determinar: Nivel o grado de escolaridad al cual ingresa el alumno; fortalezas y necesidades para proyectar flexibilización curricular o Si el alumno necesita acompañamiento en aula regular. De igual manera Definir qué profesionales podrán prestar sus servicios de prácticas, como acompañante pedagógico en aula regular, la forma o convenio institucional por parte de las universidades con las secretarías de educación distrital y municipal para tal fin.

\section{Proyecto de ley 083}

A partir del proyecto de ley 083 de (2015) Se debe asegurar que se brinden medidas efectivas basadas en las características individuales de las personas T.E.A. y condiciones similares, con el fin de maximizar el desarrollo social y la inclusión educativa. Habrá de tenerse en cuenta los diferentes grados cognitivos que se presentan dentro del Espectro del Autismo y condiciones similares, ya que se encuentran personas con nivel bajo, medio y superior a la media, así mismo, se prestarán medios de comunicación como (diapositivas, medios tecnológicos, entre otros). En caso de que la persona no cuente con una comunicación verbal.

\section{Discusión y resultados}

A partir de la información recolectada en los 30 documentos analizados, se logró apreciar los avances que se han venido desarrollando durante los últimos 10 años, en donde se inicia hablando del TEA como una enfermedad, un Síndrome o incluso categorizarla dentro de los TGD, desde la investigación se logra que el Autismo va más allá de un concepto. Es una condición de vida, donde las personas perciben el entorno social de distintas formas y por tal motivo es obligación del mismo contexto adaptarse para que logren convivir de manera asertiva.

Por tal motivo, al anteponer los diferentes puntos de vista encontrados en los documentos seleccionados, se evidencia que en el transcurso del tiempo se ha destacado significativamente el rol de la educación frente a la parte social, comunicativa y de interpretación de la población. Diseñando desde estos tres aspectos orientaciones y estrategias específicas para fortalecer las habilidades de las personas de acuerdo a las características y capacidades innatas de cada una.

De igual manera desde la recolección se evidenció que conforme iba transcurriendo la investigación, se aumentaba de manera significativa los recursos y apoyos documentales existentes frente a la educación de personas con TEA, encontrando materiales gráficos, sistemas de comunicación aumentativos y alternativos y diferentes pautas que los docentes pueden implementar en las instituciones.

Por ende, al tabular la información se obtuvo que de los 50 documentos provenientes de Colombia, el 14\% se realizaron en el periodo de 2007 a 2009, 12\% del 2010 a 2012, por otra parte el 22\% de la información se obtuvo del 2013 a 2015 y por último con un 52\% se ubica el periodo de 2016 a 2017; siendo este el más productivo en la indagación.

Con base en lo expuesto se logró evidenciar que el año más significativo para el proceso educativo de la población con TEA fue el 2017 con un 30\% de información a comparación de otros años, resaltando que los hallazgos, avances y Documentos oficiales fueron incrementando progresivamente, siendo ello de gran aporte para la Población, los docentes, familias y demás comunidad.

\section{Recolección Documental por Año}


Para finalizar, es importante resaltar la parte educativa en el desarrollo integral de la población, puesto que entorno a ella se garantiza la calidad de vida e inclusión social. Por otro lado, el MinEducación debe ejercer su papel protagónico y generar constantes avances en los lineamientos pedagógicos presentes, siendo acordes con los cambios operados desde la investigación y la educación.

Se espera en consecuencia, que lo registrado durante todo este documento, le aporte al estudio del TEA, con el fin de favorecer a la población desde el ámbito educativo, permitiendo una mayor participación, permanencia y educación de calidad; donde se vea reflejada los docentes y padres de familia en la creación de nuevas estrategias comunicativas y ambientes de aprendizaje que beneficien tanto a los estudiantes como a toda la comunidad educativa

\section{Conclusiones}

El trastorno del Espectro Autista es una condición en constantes descubrimientos y hallazgos, puesto que aún se desconocen algunos aspectos del causante, portal razón a continuación se logran evidenciar aportes significativos para la investigación de este trastorno.

En el año 2010, la (Fundación centros de Aprendizaje), estipula una serie de pautas para el trabajo con la población, señalando en alguna de ellas la eliminación de barreras de aprendizaje que se encuentran en el contexto del estudiante y la ampliación de los recursos para la construcción de conocimientos.

Posteriormente, en el año 2012, Piñeros resalta la metodología ABA como una estrategia educativa que consiste en la reducción de los comportamientos inapropiados, logrando una mejor integración del niño con su entorno. Siguiendo la misma línea, González en el año 2015, realizó una definición acerca de los materiales gráficos existentes para la población, comprendiendo la importancia del uso de dibujos, pictogramas, láminas en los diferentes escenarios (educativo, familiar, laboral).

Merece una atención especial, el año 2017, al presentar mayores adelantos referidos a la temática estudiada y a las diferentes categorías que se tuvieron en cuenta. Desde lo educativo, es importante resaltar el Decreto 1421, donde se presentan diferentes pautas y orientaciones para realizar ajustes razonables en el trabajo con la población, este se realiza de forma individual, para así lograr resaltar las capacidades y habilidades de cada uno. Durante este mismo año, se postuló el proyecto de Ley 046, desde el cual se dan pautas y estrategias específicamente para el trabajo con TEA. Estos dos documentos colombianos, logran dar respuesta a la problemática inicial planteada, sin embargo, es importante hacer partícipes a los padres de familia y demás comunidad en esta documentación.

Cómo último aporte se evidencia en el proyecto de ley 046 grandes hallazgos y metodologías importantes para lograr incluir asertivamente a la población con TEA, esto debido a que propone rutas de acción ante diferentes requerimientos como lo son ajustes razonables, acompañamiento particular y adaptación de material. Para el logro del proyecto, este mismo establece las acciones que debe realizar la secretaría de educación para así generar mayor participación de las entidades oficiales. Por tal Razón es indispensable que el congreso siga trabajando en la implementación real de una Ley que vele específicamente por la población. De igual manera de acuerdo a los resultados evidenciados se hace hincapié en seguir trabajando para realizar leyes o proyectos que apoyen a la población con TEA.

\section{Bibliografía}

Acevedo, M., Acevedo, G. L., Acosta, C. E., Acosta, A., Acosta, F., \& Adúriz, A. (2017). ¿Podemos hablar acerca del Autismo? Bogotá D.C: Magisterio.

Acevedo, M., Acevedo, G. L., Acosta, C. E., Acosta, A., Acosta, F., \& Adúriz, A. (2017). Discapacidad, Altas Capacidades Intelectuales y Trastornos del Espectro Autista. Bogotá D.C: Magisterio.

Aguilar Arevalo , A., Marin Galvis , L. A., \& Morales Verano, A. (2009). Programa de actividades para docentes de aula regular con población autista. Universidad de San Buenaventura Colombia .

Albarracín Rodríguez, A. P., Rey Hernández, L. A., \& Jaimes Caicedo, M. M. (2014). Estrategias de afrontamiento y características sociodemográficas en padres de hijos con trastornos del espectro autista. Revista Virtual Universidad Católica del Norte .

Almazán Martínez, M. E. (2009). Los sistemas aumentativos y/o alterantivos de la comunicación . Innovación y experiencias educativas

Bareño Rodríguez, C. M. (2015). Inclusión Educativa y Trastorno del Espectro Autista (TEA). Universidad Nacional de Colombia.

Beltrán, A., Guzmán, N. S., Mazo Pineda, D., Segura Sila, P., \& Tapias, M. (2016). El arteterapia como estrategia pedagógica de la educación del niño con Trastornos de Espectro del Autismo (TEA). Universidad del Atlántico.

Betancourt Loaiza, A., \& López Builes, M. A. (2017). La comprensión en el autista. Universidad de San Buenaventura Colombia.

Bravo, H. (2008). Estrategias pedagógicas y didácticas para el desarrollo de las inteligencias múltiples y el aprendizaje autónomo. Universidad del Sinú.

Bustamante, J., \& Mejia, C. (2017). Acciones y herramientas de software para implementar entornos de inclusión educativa en Colombia.

Calle Daza, S. (2017). Diseño de una herramienta didáctica para niños con trastorno del espectro autista. Universidad Pontificia Bolivariana.

Casas Rodríguez, J. P., \& Aparacio Pico , L. E. (2016). Un análisis del autismo desde la perspectiva de su influencia en familias y la tecnología como facilitador en el manejo de esta condición. Revista Logos, Ciencia y Tecnología.

Castaño González, C. (2017). Participación social de las personas con trastornos del espectro autista: tensiones, resistencias y lecciones aprendidas. Universidad Nacional de Colombia.

Castro Ortiz, M. M., \& Ñañez Girón, D. M. (2017). Estrategias pedagógicas en la educación de niños autistas (TEA). Universidad de San Buenaventura Colombia.

Cifuentes Tarazona, L. Y. (2017). Las tecnologías de información y la comunicación (TIC) como oportunidad para fortalecer la intencionalidad comunicativa oral en estudiantes con autismo de ciclo 1. Universidad de la Sabana.

Congreso de Colombia. (2007). Ley 1145

Congreso de Colombia. (2013). Ley Estatutaria 1618.

Congreso de la Republica. (2017). Proyecto de Ley número 046. El autor.

Congreso de la República de Colombia . (2017). Informe de ponencia para segundo debate del proyecto de ley 046 de 2017.

Congreso de la república de Colombia. (1994). Ley General de Educación 115.

Cortes Briceño , D. L. (2016). Escuelas Diversas: inclusión escolar de niñas, niños y jóvenes con autismo en Bogotá. Universidad Libre.

Coy Guerrero, L., \& Martín Padilla , E. (2017). Habilidades sociales y comunicativas a través del arte en jóvenes con trastorno del espectro autista (TEA). Estudios Pedagógicos

Díaz Marentes, S. M., \& Alvarez Chaparro, M. (2016). Identificación de aspectos que influyen en el acceso, transito y socialización de estudiantes con autismo, en la educación superior en la ciudad de Bogotá. Universidad Piloto de Colombia. 


\section{La educación de las personas con trastorno del espectro autista}

\section{Colombia en los últimos 10 años}

Fundación Centros de Aprendizaje . (2010). Orientaciones pedagógicas para la inclusión de estudiantes en condición de discapacidad.

Garzón Guesgüan., L. F., \& Sanchez Alfonso., W. E. (2016). La pintura como estrategia pedagógica para mejorar la comunicación en la población con TEA de la fundación CERES en Bogotá Colombia. Corporación Universitaria Minuto de Dios.

Giraldo Torres, L. R., Restrepo de Mejía, F., \& Arboleda Sánchez, V. A. (2017). Trastorno del espectro autista, electroencefalografía y neuronas espejo. Acta Neurológica Colombiana.

González Moreno , C. X. (2017). El juego como estrategia para el desarrollo del lenguaje en un niño con trastorno del espectro autista desde el ámbito de la educación inclusiva. Revista de investigación educativa de la rediech.

González, M. (2015). Trastorno del espectro autista: una perspectiva pedagógica dirigida al profesorado. Obtenido de Trastorno del espectro autista: una perspectiva pedagógica dirigida al profesorado.

Grisales Castaño, L. V. (2015). Los trastornos del espectro autista y su intervención. Implementación de mecanismos de inclusión escolar por parte de la fundación Prisma. Diseño de un modelo de sistematización de experiencias en la EPIS. Universidad del Valle.

Gutiérrez Ruiz , K. (2016). Identificación temprana de trastornos del espectro autista. Acta Neurológica Colombiana .

Instituto Colombiano de Bienestar Familiar ( ICBF). (2010). Orientaciones pedagógicas para la atención y la promoción de la inclusión de niñas y niños con autismo.

Instituto de Evaluación Tecnológica en Salud. (2014). Terapias de análisis de comportamiento aplicado ABA, para el tratamiento de personas con diagnóstico de trastornos del espectro autista y trastorno de hiperactividad y déficit de atención .

Leo, K. (1943). Trastornos autistas del contacto afectivo. Revista Española de Discapacidad Intelectual .

López Serna, I. L., Ocampo Muñoz, M. A., \& Peña Valencia, J. C. (2016). Experiencias sobre la escolarización de niños con trastorno del espectro autista, desde la perspectiva de padres, docentes y directivas. Universidad del Valle.

Mariño Villamizar, Y. A. (2017). Implementación de estrategias pedagógicas para niños y niñas con trastorno del espectro autista, hogar comunitario Tribilin,Bucaramanga,Santander. Corporación Universitaria Minuto de Dios.

MinEducación. (2006). Orientaciones pedagógicas para la atención educativa a estudiantes con Autismo. Obtenido de Orientaciones pedagógicas para la atención educativa a estudiantes con Autismo.
MinEducación. (2009). Decreto 366. 7.

MinEducación. (2017). Decreto 1421.

MinEducación. (2017). Decreto 1421.

MinEducación. (2017). PIAR ( Plan Individual de Ajsutes Razonables).

MINSALUD. (2015). Protocolo clínico para el diagnóstico, tratamiento y ruta de atención integral de niños y niñas con Trastornos del Espectro Autista.

Mira Pastor, R., \& Grau, C. (2017). Los sistemas alternativos y aumentativos de comunicación (SAAC) como instrumento para disminuir conductas desafiantes en el alumnado con TEA: estudio de un caso. Revista Española de Discapacidad.

Morales Sandoval , M. C. (2017). Estudios jurídicos de garantías fundamentales en caso de niños en situación de discapacidad autismo - Acceso a la salud como primera medida para garantizar un ejercicio completo de garantías constitucionales. Universidad Católica de Colombia .

Ortega Cuartas , E. G. (2015). Percepción de la inclusión de niños con trastorno del espectro autista (TEA) desde las voces y vivencias de los docentes a partir de las políticas educativas. Universidad de Manizales.

Paredes Quiñonez , F. J. (2012). Propuesta para la enseñanza de la comunicación aumentativa y alternativa en la población con trastorno del espectro autista. Universidad Pedagógica Nacional de Colombia.

Pérez Rivero, P. F., \& Martínez Garrido , L. (2014). Perfiles cognitivos en el trastorno autista de alto funcionamiento y el Síndrome de Asperger. Revista CES Psicología.

Piñeros Ortiz , \& Toro Herrera. (2012). Conceptos generales sobre ABA en niños con trastorno del espectro autista. Revista Fac Med.

Proyecto de Ley 083. (2015). 33.

Rodríguez Romero , L., \& Mariño Ruiz, M. C. (2017). Estudio de caso independencia y funcionalidad en niño con autismo de 5 años. Universidad Politécnico Grancolombiano .

Vaillancourt, G. (2012). Música y Musicoterapia. Su importancia en el desarrollo infantil. Madrid (es): Narcea.

Wilches Rache, K. J. (2015). Inclusion social y educativa de los trastorno espectro autista (TEA). Universidad Nacional Abierta y a Distancia UNAD.

Wing, L. (1998). El Autismo en niños y adultos. Barcelona: Paidos. 